\title{
A comparative analysis of five cropland datasets in Africa
}

\author{
WEI Yanbing ${ }^{12}$, LU Miao $^{12} *$ WU Wenbin ${ }^{12}$
}

1. Key Laboratory of Agricultural Remote Sensing, Ministry of Agriculture, Beijing 100081, P.R.China

2. Institute of Agricultural Resources and Regional Planning, Chinese Academy of Agricultural Sciences, Beijing 100081, P.R.China

KEY WORDS: Africa, Land cover, Cropland area, Spatial location, Accuracy

\begin{abstract}
:
The food security, particularly in Africa, is a challenge to be resolved. The cropland area and spatial distribution obtained from remote sensing imagery are vital information. In this paper, according to cropland area and spatial location, we compare five global cropland datasets including CCI Land Cover, GlobCover, MODIS Collection 5, GlobeLand30 and Unified Cropland in circa 2010 of Africa in terms of cropland area and spatial location. The accuracy of cropland area calculated from five datasets was analyzed compared with statistic data. Based on validation samples, the accuracies of spatial location for the five cropland products were assessed by error matrix. The results show that GlobeLand30 has the best fitness with the statistics, followed by MODIS Collection 5 and Unified Cropland, GlobCover and CCI Land Cover have the lower accuracies. For the accuracy of spatial location of cropland, GlobeLand30 reaches the highest accuracy, followed by Unified Cropland, MODIS Collection 5 and GlobCover, CCI Land Cover has the lowest accuracy. The spatial location accuracy of five datasets in the Csa with suitable farming condition is generally higher than in the Bsk.
\end{abstract}

\section{INTRODUCTION}

The extent, distribution characteristics of cropland are fundamental information which have long been identified as significant influences on global food security, climate change and environmental sustainable development (Jayne et al., 2014a). The food security particularly in Africa is still a challenge to humankind. It is estimated that food demand is expected to increase more than $60 \%$ in Africa by 2050 compared with $2005 / 2007$, if the situation of cropland does not change, only few countries are able to be self-sufficiency (Martin et al., 2016a). The exact cropland area and spatial distribution are vital information to the study of food security which cannot be obtained by statistic data.

Because of the consistently and efficiently monitoring on temporal and spatial scale, Satellite data has becoming a major reference for cropland mapping (Russell et al., 2014a). Since 2000, global land cover datasets from high resolution satellite sensors have becoming popular. MODIS Collection 5 product made by Boston University was derived from the MODIS data of 2000-2012 with 500m spatial resolution (Friedl et al., 2010a). ESA (European Space Agency) produced the GlobCover land cover dataset of 2005 and 2009 with 300 m resolution by using ${ }^{1}$ MERIS (Medium Resolution Imaging Spectrometer) reflection data (Bicheron et al., 2008a; Bontemps et al., 2011a). Aiming to deal with climate issue in 21 century, CCI Land Cover was produced by ESA, based on MERISFR data and updated by SPOT-VGT data (Defourny et al., 2014a). Unified Cropland dataset with $250 \mathrm{~m}$ resolution in circa 2014 was derived from existing global land cover maps (François et al., 2016a). NGCC (National Geomatics Center of China) produced GlobeLand30 dataset with $30 \mathrm{~m}$ spatial resolution by using TM data and ETM+ data as basis and HJ-1 dataset as reference (Chen et al., 2015a).Varieties of satellite sensors, spatial resolutions, classification schemes and mapping technologies result in the difference between global land cover datasets (Pérez-Hoyos et al., 2012a). For product users and producers, clarifying the strengths and weaknesses of datasets is vital. In recent years, several researchers have compared the difference and consistency of global land cover datasets. Commonly existing methods for the comparison can be classified into two categories: assessing classification data derived from global land cover datasets according to the comparison with statistic data (Pérez-

\footnotetext{
* Corresponding author - lumiao0616@163.com
} 
Hoyos et al., 2012a; Wu et al., 2009a; Steffen Fritz et al., 2010a); using error matrix to estimate the accuracy of spatial location for global land cover products with validation samples (Wickham et al., 2010a; Lu et al., 2016a; Yang et al, 2014a). However these studies focus on global land cover datasets with coarse spatial resolution, such as UMD, IGBP-DISCover and GLC2000, lack the comparison of global land cover datasets with high resolution in recent years. In addition, few studies assessed the spatial location accuracy by the validation samples, which can reduce the error provided by the reference data.

Focusing on the cropland in Africa, the objective of this study is to compare cropland datasets derived from five global land cover

\section{DATA RESOUCES AND PRECESSING}

\subsection{Data Resources}

Table 1 shows the information of the five global land cover datasets. Based on the UN-LCCS (United Nations Land Cover Classification System), GlobCover2009 divided the global land cover into 22 types by using the combination of supervised and unsupervised classification (Bontemps et al., 2011), CCI Land Cover used unsupervised and supervised classification to obtain 22 major types and 16 secondary types at the global scale (Defourny et al., 2014; Wei et al., 2016a). According to POKbased (pixel-object-knowledge-based) classification, GlobeLand30 sort global land cover into 10 types (Chen et al., datasets, including MODIS Collection5, GlobCover, GlobeLand30, CCI Land Cover and Unified Cropland. To measure the accuracies of cropland area, the five cropland datasets are used to compute the cropland area at country level and then compare the cropland area with the statistic data. According to the validation samples, the overall accuracies and Kappa coefficients of five datasets were calculated to evaluate the accuracies of spatial location by error matrix. This study shows the strengths and shortages of five global land cover products in Africa, which provides a reference for the product users to choose the appropriate cropland datasets for their researches, and helps the product producers to improve the methodology and technology of data processing in the future.

2015a; Brovelli et al., 2015a). Following IGBP classification, MODIS Collection used decision tree classification to sort the land cover into 17 types (Friedl et al., 2010a). Unified Cropland is derived from three datasets by mean value resampling, every value of pixel represents the cropland proportion (François et al., 2016).

The supplementary data in 2010 including cropland statistic area and climate classification in Africa. The cropland area is sourced from FAO statistic http://www.fao.org. Based on the proportion of cropland in African countries provided by FAO, the cropland area can be calculated. KÖPPEN-Geiger climate classification divides Africa into 13 climate zones (Peel et al., 2007a), the vector data are available to download on the website of WORLD MAPS OF KÖPPEN-GEIGER CLIMATE CLASSIFICATION.

\begin{tabular}{ccccc}
\hline Product & Time & Satellite sensor & $\begin{array}{c}\text { Spatial } \\
\text { resolution }\end{array}$ & Cropland class \\
GlobCover & 2009 & ENVISAT/MERIS & $300 \mathrm{~m}$ & $\begin{array}{c}\text { Irrigated croplands, rained croplands, mosaic cropland (50- } \\
70 \%) / \text { vegetation }(20-50 \%) \text {, mosaic vegetation }(50-\end{array}$ \\
& & & & 70\%)/cropland $(20-50 \%)$
\end{tabular}

Table 1. Characteristics of five cropland datasets used in this study 


\subsection{Data Preprocessing}

Due to the difference between the spatial resolution and classification, data preprocessing is indispensable before the comparison of five global land cover datasets. First of all, the projects of the five global land cover datasets were transformed into WGS-84 by using nearest-neighbor resampling. Because this paper is focusing on the cropland in Africa, it is necessary to harmonize the cropland classification of five datasets. According to the definition of croplands provided by the FAO, the classification related to the cropland were fused (Table 1). Both of GlobeLand30 and Unified Cropland only include one kind of cropland. MODIS Collection5 includes both of cropland and mosaic cropland / vegetation. GlobCover and CCI Land Cover include four types of cropland, GlobCover contains irrigated croplands, rained croplands, mosaic cropland (50$70 \%) /$ vegetation $(20-50 \%)$ and mosaic vegetation (5070\%)/cropland (20-50\%). CCI Land Cover contains Rain croplands, irrigated croplands, mosaic cropland $(>50 \%) /$ vegetation $(<50 \%)$, mosaic vegetation $(>50 \%) /$ cropland $(<50 \%)$. MODIS Collection5 has the coarsest spatial resolution among five datasets, the other four datasets were normalized to the resolution of $500 \mathrm{~m}$ by using the method of nearest neighbor resampling. On the basis of the vector data of Africa's boundary, five products were clipped to obtain the cropland datasets in Africa. Finally, the cropland proportion in each pixel was calculated. The five cropland datasets of China after preprocessing were shown in Figure 2.
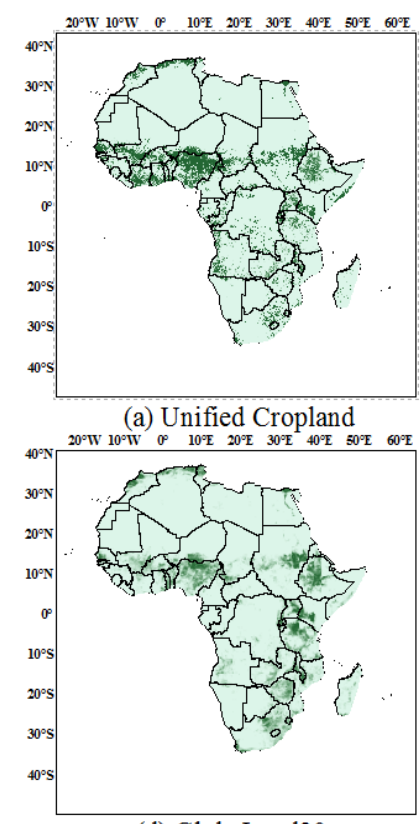

(d) GlobeL and30
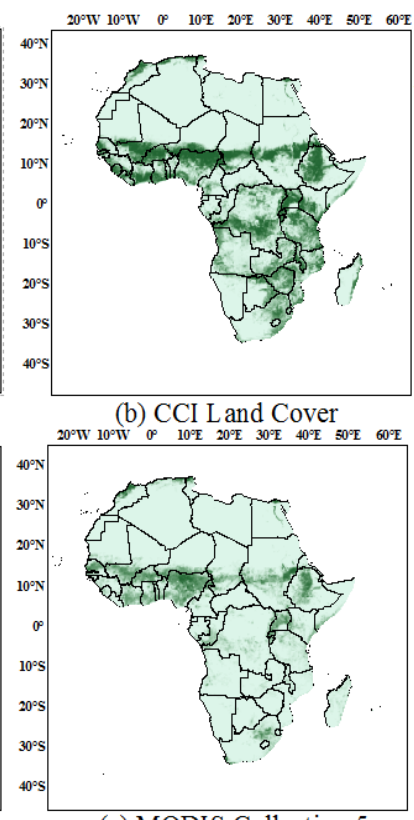

(e) MODIS Collection 5

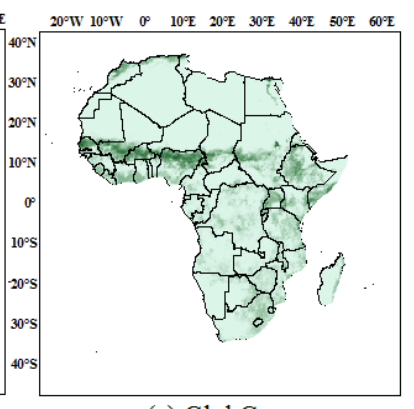

(c) GlobCover

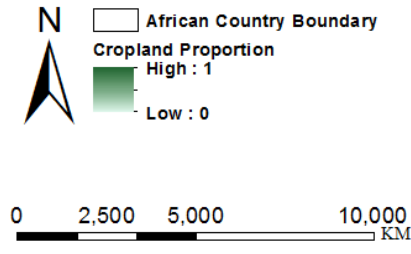

Figure 2. Five cropland datasets after preprocessing

\section{METHOLOGY}

In this paper, the comparison of five cropland datasets in Africa including the accuracies of datasets in cropland area and spatial location. In terms of cropland area, compared with statistic data, the accuracies of five cropland datasets were estimated at country and continent scale. In terms of spatial location, the overall accuracies and Kappa coefficients were calculated to assess the accuracies of five cropland datasets, based on validation samples. According to the climate classifications in
Africa, the overall accuracies was computed in different climate zones.

\subsection{Cropland Area Comparison}

Based on the African country boundary, the cropland area was calculated from five cropland datasets, then the cropland area of African continent was summarized. On the basis of the cropland area of African countries in 2010 from FAO, the root mean square error (RMSE) between the statistic data and the cropland derived from five datasets was computed to represent the 
dispersion between statistic data and five cropland datasets. The smaller the RMSE is, the smaller dispersion will be, the RMSE was computed as follows:

RMSE $=\sqrt{\frac{\sum_{i=1}^{n}\left(x_{i}-y_{i}\right)^{2}}{n}}$

Where $x_{i}$ is the cropland area in country $\mathrm{i}$, calculated from five datasets, $y_{i}$ is the cropland area from statistic data in country i, $\mathrm{n}$ is the number of African countries, the correlation coefficient was computed to reflect the correlation between statistic data and cropland datasets, the higher the correlation coefficient is, the higher correlation will be, the correlation coefficient was computed as follows:

$\mathrm{R}=\frac{\sum_{i=1}^{n}\left(x_{i}-\bar{x}\right)\left(y_{i}-\bar{y}\right)}{\sqrt{\sum_{i=1}^{n}\left(x_{i}-\bar{x}\right)^{2} \cdot \sum_{i=1}^{n}\left(y_{i}-\bar{y}\right)^{2}}}$

Where $\bar{x}$ is the average cropland area from datasets in African countries, $\bar{y}$ is the average cropland area of statistic data.

\subsection{Spatial Location Comparison}

Only assessing the global land cover in cropland area exists limitation, the cropland area accuracy cannot describe anything about the spatial accuracy of cropland. Thus, assessing the accuracy of spatial location is necessary (Wu et al., 2009a). The conventional method prefer to select a global land cover with high accuracy as reference data, then compare the spatial difference between the target products and reference data. While the shortage of this method is extremely relying on the reference data, the inaccuracy of the reference data might lead to the error of finally result (Li et al., 2010a; Pflugmacher et al., 2011a). In this paper, the validation samples were selected as reference, the spatial location accuracy was assessed basing on this reference data.

The quality, quantity and distribution of the validation samples have been identified as the vital influence on the result of accuracy assessment (Foody, 2010a). In this study, one part of the validation samples is derived from FROM-GLC (Gong et al., 2013a; Yu et al., 2014a), the global land cover was parted into 7000 hexagons with equal area, 5 samples were selected randomly in each hexagon to ensure the well-distributed of the samples, then the classes of the samples were identified by visual interpretation to ensure the correctness of the classification. However, there are 431 cropland samples and 6364 non-samples in Africa, the wide gap between the number of cropland and samples cannot satisfy the demand of the accuracy assessment. Hence, samples were selected randomly basing on the cropland distribution of five datasets. According to the Google Earth imagery, 650 cropland samples were verified. The two sources samples were gathered to be the validation samples of five datasets, with 1081 cropland samples and 6364 non-cropland samples, as shown in Figure 3. Based on these validation samples, the confusion matrix was used to compute the overall accuracy and Kappa coefficient to compare the accuracy of spatial location.

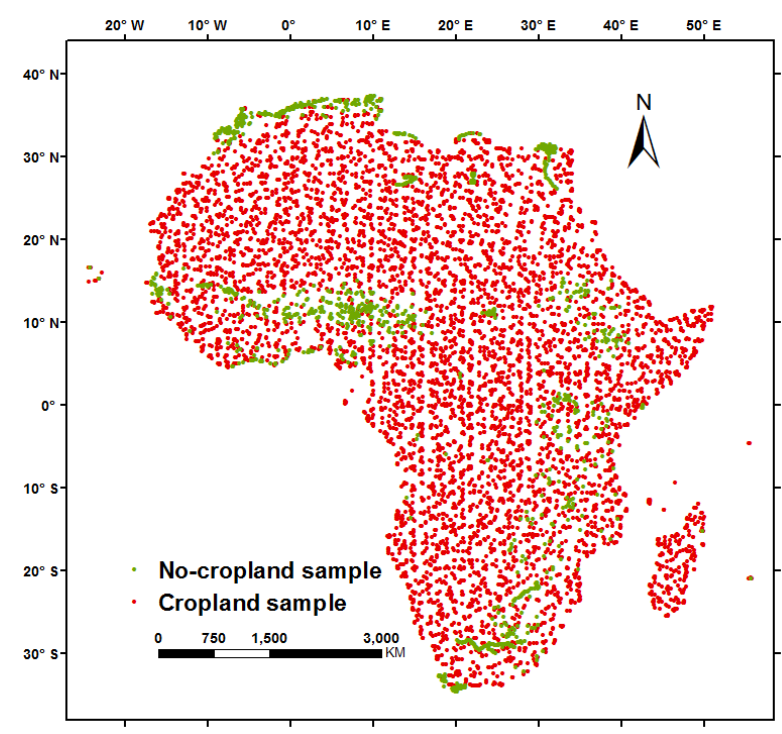

Figure 3. Spatial distribution of the validation samples

Africa is divided into 13 zones by KÖPPEN-Geiger climate classification (Peel et al., 2007a): Hot-summer Mediterranean climate (Csa), Subtropical highland climate (Cwb), Temperate mediterranean climate(Csb), Cold semi-arid climate(Bsk), Equatorial climate(Af), Tropical savanna climate(Aw), Hot semi-arid climate(Bsh), Temperate oceanic climate(Cfb), Hot desert climate(Bwh), Monsoon climate(Am), Humid subtropical climate(Cfa), Monsoon-influenced humid subtropical climate(Cwa), Cold desert climate(Bwk). Basing on these climate classifications, we select 5 climate zones where the cropland are mainly distributed to evaluate the spatial accuracies of five datasets in these zones. 


\section{RESULT}

\subsection{Comparison of Cropland}

The various differences can be found between the cropland areas computed from five cropland datasets and statistic data in each African country. CCI-LC is generally overestimated the cropland area, the cropland areas derived from GlobeLand 30 and MODIS Collection5 are close to the statistic with small gaps. We compared the arable area in Africa summarized by the five cropland datasets and statistic data (Figure 4). Overall, all of five maps overestimate the cropland area, the cropland area calculated from GlobeLand 30 is $23.4 \times 10^{5} \mathrm{~km}^{2}$, which is the closest to the statistics by $19.6 \times 10^{5} \mathrm{~km}^{2}$. Followed by the cropland area derived from MODIS Collection5 and GlobCover, which are $25.4 \times 10^{5} \mathrm{~km}^{2}$ and $26.4 \times 10^{5} \mathrm{~km}^{2}$. Compared with statistic area, Unified Cropland and CCI-LC have a greater degree of overestimation.

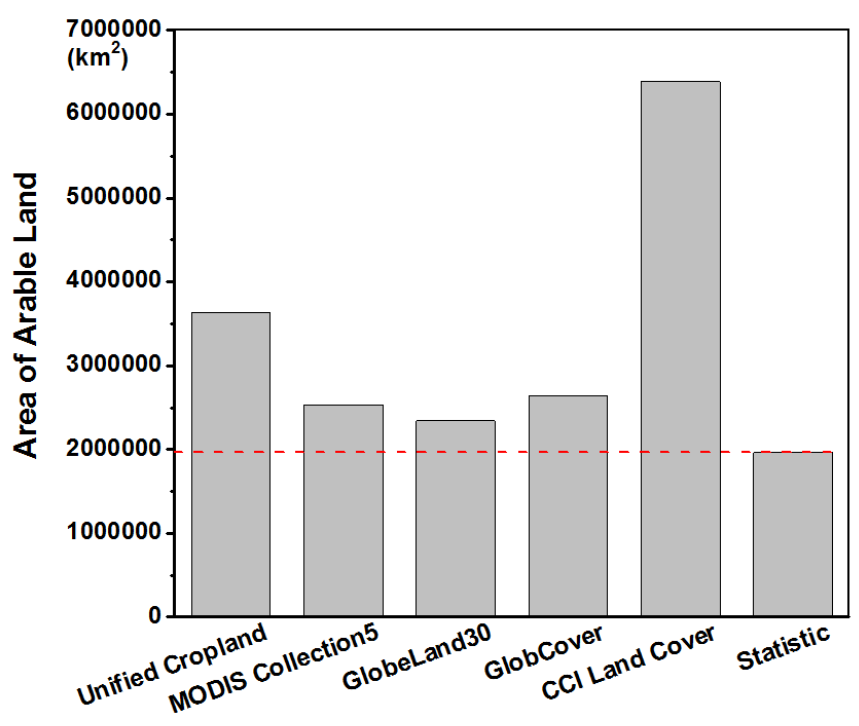

Figure 4. Total cropland area in Africa from statistics and cropland datasets
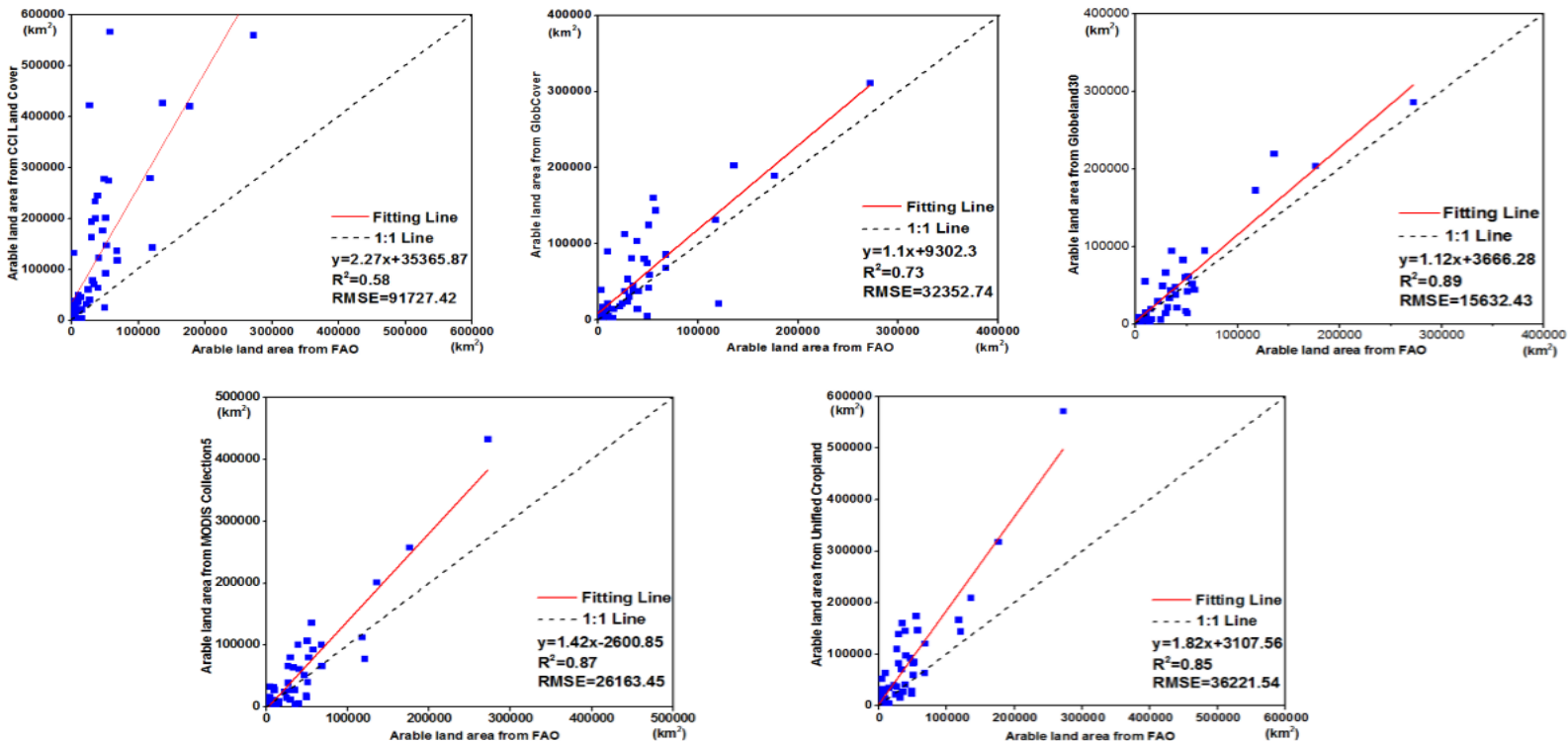

Figure 5. Dispersion and correlation of the area between the cropland dataset and statistic

RMSE and correlation coefficients were computed to show the dispersion and fitness between the five cropland datasets and statistic data (Figure 5). The solid line reflects the trend between cropland dataset and statistics. The RMSE value of GlobeLand30 is smaller than others, with a data distribution on or near the 1:1 line, which reflects the least dispersion from the statistic data. The RMSE value of MODIS Collection5 is also comparatively small, the data points of MODIS Collection 5 are distributed near 1:1 line, with a large gap of the minority. CCILC has the largest dispersion with statistic data, by the highest RMSE value and a scattered distribution with MODIS Collection5, Unified Cropland, GlobCover, CCI-LC and the statistic data are $0.89,0.87,0.85,0.73$ and 0.58 . The higher the value of correlation is, the better fitness with statistic will be. 
The best fitness was obtained from GlobeLand30, followed by MODIS Collection 5. The poorest fitness was between CCI-LC and statistic data. Generally the lowest dispersion and best fitness can easily be found from GlobaLand30, which reflect an accurate result of cropland area in African countries. Furthermore, this paper also found that the monitoring effect of

\subsection{Comparison of the Spatial Location}

The Kappa coefficient and overall accuracy were calculated via confusion matrix to assess the spatial location accuracy of five cropland datasets (Table 6). GlobeLand30 has the best accuracy
MODIS Collection 5 with $500 \mathrm{~m}$ resolution is better than Unified Cropland with $250 \mathrm{~m}$ resolution and GlobCover, CCI-LC datasets with $300 \mathrm{~m}$ resolution, implying that the low spatial resolution data can also obtain the accurate monitoring result by appropriate classification approach and method.

of spatial location, with the highest overall accuracy and Kappa coefficient, by $90.17 \%$ and 0.62 . Followed by the Unified Cropland and MODIS Collection, the overall accuracies and Kappa coefficients are comparatively high, by $88.72 \%$ and 0.6 , $88.17 \%$ and 0.54 , respectively. The lower accuracies of spatial location were obtained from GlobCover and CCI-LC, with the poor overall accuracies and Kappa coefficient.

\begin{tabular}{cccccc}
\hline & CCI Land Cover & GlobCover & GlobeLand30 & MODIS Collection5 & Unified Cropland \\
\hline Overall accuracy/\% & 79.83 & 85.08 & 90.71 & 88.17 & 88.72 \\
Kappa coefficient & 0.43 & 0.47 & 0.62 & 0.54 & 0.60 \\
\hline
\end{tabular}

Table 6 . The spatial location accuracies of the five cropland datasets

Aiming to analysis the accuracies of spatial location in various climate zones, 5 climate zones were selected to assess accuracies by overall accuracies, including Tropical savanna climate(Aw), Hot semi-arid climate(Bsh), Cold semi-arid climate(Bsk), Hotsummer mediterranean climate(Csa) and Subtropical highland climate $(\mathrm{Cwb})$, as shown in Figure 7 . In the Bsk, the best accuracy is $81.16 \%$, which obtained from Unified Cropland, CCI-LC has the lowest overall accuracy of $71.16 \%$. In the Csa, GlobCover achieves the highest accuracy of $81.16 \%$. GlobeLand30 provides the best accuracies in the Aw, Bsh and Cwb, by $88.25 \%, 84.55 \%$ and $85.91 \%$, respectively. On the contrary, the overall accuracies of CCI-LC are relatively low in these three zones, corresponding to $65.81 \%, 71.98 \%$ and $66.44 \%$. Above all, in five climate zones, the accuracies of five datasets are higher in the Csa and Aw, and comparatively low in the Bsk. Among five cropland datasets, the location accuracies of GlobeLand30 are greater in each climate zone, while the relatively low accuracies were obtained from CCI-LC.

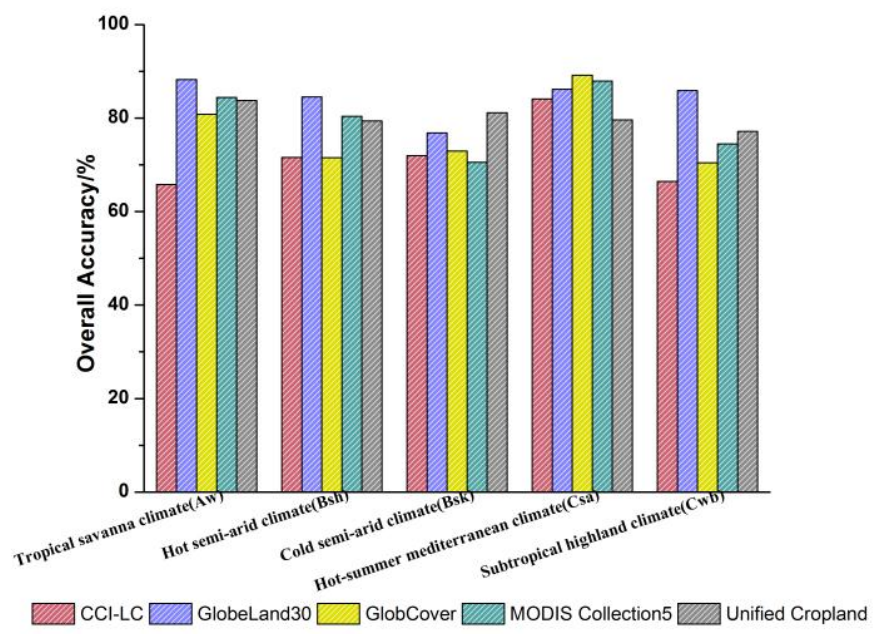

Figure 7. Overall accuracies of the five cropland datasets in five climate zones 


\section{CONCLUSION}

This paper took African cropland as the research object, compared five global cropland datasets in circa 2010, including Unified Cropland, MODIS Collection 5, GlobeLand30, GlobCover, CCI Land Cover, according to the assessment of cropland area, spatial location. The result shows that, GlobeLand30 has the highest accuracies of cropland area, with the lowest dispersion and best fitness. Followed by MODIS Collection 5 and Unified Cropland, the relatively low accuracies are obtained by GlobCover and CCI-LC. For the accuracies of spatial location, GlobeLand30 also reaches the highest accuracy, followed by the Unified Cropland, MODIS Collection 5 and GlobCover. CCI-LC achieves the poorest accuracy than other four cropland datasets. Comparing the spatial location accuracy in 5 climate zones, the higher overall accuracies can be found in the Csa which has perfect farming condition with intensive distribution of cropland. While values of accuracy obtained from five datasets are generally low in the Bsk with a scattered distribution of cultivated land. It can be concluded that the place with well climate condition has better spatial location, the cropland can be distinguished easily by five datasets than the place with a poor condition for planting.

In general, dataset with higher spatial resolution has greater mapping accuracy. However this paper found this conclusion is not completely true. MODIS Collection 5 dataset with the lowest resolution in five products had a better fitness with statistics than others, expect GlobeLand30 dataset. The overall accuracy and Kappa coefficient of MODIS Collection are higher than the value of GlobCover and CCI-LC. It can be concluded that not only should the spatial resolution of remote sensing data be concerned when selecting the datasets, but its classification system and classification method are also the keys to influence the mapping precision. Low spatial resolution dataset can also obtain the accurate monitoring result by appropriate classification approach and method.

\section{REFERENCES}

Bicheron P, Defourny P, Brockmann C, Schouten L, Vancutsem C, Huc M, Bontemps S, Leroy M, Achard F, Herold M, Ranera
F, Arino O, 2008. GlobCover Products Description and Validation Report.

Brovelli Antonia Maria, Monia Elisa Molinari, Eman Hussein, Jun Chen, Ran Li, 2015a. The First Comprehensive Accuracy Assessment of GlobeLand30 at a National Level: Methodology and Results. Remote Sensing, 7: 4191-4212.

Bontemps S, Defourny P, Bogaert E V, Arino O, Kalogirou V, Perez J R, 2011. GLOBCOVER 2009 Products Description and Validation Report.

Chen J, Chen J, Liao A P, Cao X, Chen L J, Chen X H, He C Y, Han G, Peng S, Lu M, Zhang W, Tong X H, Mills J, 2015a. Global land cover mapping at $30 \mathrm{~m}$ resolution: A POK-based operational approach. ISPRS-Journal of Photogramm Remote Sensing, 103: 7-27.

Defourny, P., Kirches, G., Brockmann, C., Boettcher, M., Peters, M., Bontemps, S., Lamarche, C., Schlerf, M., Santoro, M, 2014. Land Cover CCI PRODUCT USER GUIDE VERSION 2.

François Waldner, Steffen Fritz, Antonio Di Gregorio, Dmitry Plotnikov, Sergey Bartalev, Nataliia Kussul, Peng Gong, Prasad Thenkabail, Gerard Hazeu, Igor Klein, Fabian Löw, Jukka Miettinen, Vinay Kumar Dadhwal, Céline Lamarche, Sophie Bontemps and Pierre Defourny, 2016a. A Unified Cropland Layer at $250 \mathrm{~m}$ for Global Agriculture Monitoring. Data, 1, 3.

Foody G M, 2010a. Assessing the accuracy of land cover change with imperfect ground reference data. Remote Sensing Environment, 114: 2271-2285.

Friedl M A, Sulla-Menashe D, Tan B, Schneider A, Ramankutty N, Sibley A, Huang X, 2010a. MODIS Collection 5 global land cover: Algorithm refinements and characterization of new datasets. Remote Sensing Environment, 114: 168-182.

Gong P, Wang J, Yu, L, Zhao Y C, Zhao Y Y, Liang L, Niu Z G, Huang X M, Fu H H, Liu S, Li C C, Li X Y, Fu W, Liu C X, Yue X, Wang X Y, Cheng Q, Hu L Y, Yao W B, Zhang H, Zhu P, Zhao Z Y, Zhang H Y, Zheng Y M, Ji L Y, Zhang Y W, Chen H, Yan A, Guo J H, Liang Y, Wang L, Liu X J, Shi T T, Zhu M H, Chen Y L, Yang G W, Tang P, Xu B, Giri C, Clinton N, Zhu Z L, Chen J, Chen J, 2013a. Finer resolution observation and monitoring of GLC: first mapping results with Landsat TM and ETM+ data. 
International Journal of Remote Sensing, 34: 2607-2654.

Li B B, Fang X Q, Ye Y, Zhang X Z, 2010a. Accuracy assessment of global historical cropland datasets based on regional reconstructed historical data-A case study in northeast china. Science China: Earth Sciences, 40(8):1048-1059.

Lu M, Wu W B, Zhang L, Liao A P, Peng S, Tang H J, 2016a. Science China: Earth Sciences, 11: 1459-1471.

Martin K. van Ittersum, Lenny G. J. van Bussel, Joost Wolf, Patricio Grassini, Justin van Wart, Nicolas Guilpart, Lieven Claessens, Hugo de Groot, Keith Wiebe, Daniel Mason-D’Croz, Haishun Yang, Hendrik Boogaard, Pepijn A. J. van Oort, Marloes P. van Loon, Kazuki Saito, Ochieng Adimo, Samuel Adjei-Nsiah, Alhassane Agali,Abdullahi Bala, Regis Chikowol, Kayuki Kaizzim, Mamoutou Kouressy, Joachim H. J. R. Makoi,Korodjouma Ouattara, Kindie Tesfaye, and Kenneth G. Cassman, 2016a. Can sub-Saharan Africa feed itself? PNAS 113: 14964-14969.

Peel M. C., Finlayson B. L., McMahon T. A., 2007a. Updated world map of the KÖPPEN-Geiger climate classification. Hydrology and Earth System Sciences, 11: 1633-1644.

Pérez-Hoyos A, García-Haro F.J, San-Miguel-Ayanz J, 2012a.Conventional and fuzzy comparisons of large scale land cover products: Application to CORINE, GLC2000, MODIS and GlobCover in Europe. ISPRS-J Photogramm Remote Sensing, 74: 185-201.

Pflugmacher D, Krankina O N, Cohen W B, Friedl M A, SullaMenashe D, Kennedy R E, Nelson P, Loboda T V, Kuemmerle T, Dyukarev E, Elsakov V, Kharuk V I, 2011a. Comparison and assessment of coarse resolution land cover maps for northern Eurasia. Remote Sensing Environment, 115: 3539-3553.

Russell G. Congalton, Jianyu Gu, Kamini Yadav, Prasad Thenkabail, Mutlu Ozdogan, 2014a. Global Land Cover Mapping: A Review and Uncertainty Analysis. Remote Sensing 6, 12070-12093.

Jayne, T.S., Chamberlin, J., Headey, D.D., 2014a. Land pressures, the evolution of farming systems, and development strategies in Africa: a synthesis. Food Policy 48, 1-17.
Wei Li, Philippe Ciais, Philippe Ciais, Natasha MacBean, Shushi Peng, Pierre Defourny, Sophie Bontemps, 2016a. Major forest changes and land cover transitions based on plant functional types derived from the ESA CCI Land Cover product. International Journal of Applied Earth Observation and Geoinformation, 47: 30-39.

Wickham J D, Stehman S V, Fry J A, Smith J H, Homer C G, 2010a. Thematic accuracy of the NLCD 2001 land cover for the conterminous United States. Remote Sensing of Environment, 114: 1286-1296.

Wu W B, Yang P, Zhang L, Tang H J, Zhou Q B, Ryosuke S, 2009a. Accuracy assessment of four global land cover datasets in China (in Chinese). Trans Chin Soc Agric Eng, 25: 167-173.

Steffen Fritz, Linda See \& Felix Rembold, 2010a. Comparison of global and regional land cover maps with statistical information for the agricultural domain in Africa. International Journal of Remote Sensing, 31: 2237-2256.

Yang Y K, Xiao P F, Feng X Z, LI H X, Chang X, Feng W D, 2014a. Comparison and assessment of large-scale land cover datasets in China and adjacent regions. Journal of Remote Sensing, 18:453-475.

Yu L, Wang J, Li X, Li C, Zhao Y, Gong P, 2014a. A multiresolution global land cover dataset through multisource data aggregation. Science China: Earth Sciences, 44(8): 1646-166. 